\title{
ENHANCING THE SUNSCREEN EFFICACY OF BEMOTRIZINOL MICROPIGMENT BY USING 0/W NANOEMULSION TOPICAL PREPARATIONS
}

\author{
DINA M. HASHIM1, NERMIN M. SHETA2, VIVIAN S. ELWAZZAN1, WEDAD SAKRAN³
}

1Pharmaceutics Department, National Organization for Drug Control and Research (NODCAR), Giza, Egypt, ${ }^{2}$ Pharmaceutics Department, Faculty of Pharmacy, October 6 University, Giza, Egypt, ${ }^{3}$ Pharmaceutics Department, Faculty of Pharmacy, Helwan University, Ein Helwan, Egypt

Email: dina_medhat_88@hotmail.com

Received: 17 Feb 2019 Revised and Accepted: 17 May 2019

\begin{abstract}
Objective: Bemotrizinol (BEMT) is the most efficient broad-spectrum UV-absorber having a dual mechanism of action in absorbing and reflecting photons. The main objective of this work was to develop successful oil in water $(\mathrm{o} / \mathrm{w})$ nanoemulsion for improving the solubility of BEMT and its protective characteristics.

Methods: Pseudo-ternary phase diagrams were constructed using labrafac PG and isopropyl myristate as oil phase, tween 80 as surfactant (S) and cremophor EL as cosurfactant $(\mathrm{CoS})$ the ratio of S/CoS was determined according to highest percent of water incorporation to the system. Full factorial study design $\left(2^{4}\right)$ using Design-Expert ${ }^{\circledR}$ software was adopted to study the effect of four independent variables namely: oil type, oil concentration, $\mathrm{S} / \mathrm{CoS}_{\operatorname{mix}}(3: 1)$ concentrations and BEMT concentration on the particle size and the in vitro release at $2 \mathrm{~h}\left(\mathrm{Q}_{2 \mathrm{~h}}\right)$ of the prepared nanoemulsion formulae. Two systems each of eight formulae were developed and evaluated through droplet size analysis, zeta potential measurement, refractive index, in vitro drug release and according to the desirability value two formulae (F6 and F14) were used for further evaluations including in vitro sun protection factor (SPF), ex-vivo deposition by tape stripping technique, permeation test and photostability study.
\end{abstract}

Results: Formula (F14) was chosen as the optimum formula having an in vitro SPF of $16.08 \pm 0.39$, lowest permeation of $140 \pm 0.06 \mu \mathrm{g} / \mathrm{cm}^{2}$ after six $\mathrm{h}$ and highest photostability $\left(\mathrm{t}_{90 \%}=168.02\right)$ after $120 \mathrm{~min}$.

Conclusion: Despite the poor solubility of bemotrizinol, it could be enhanced by novel drug delivery systems with good SPF value while maintaining its photostability.

Keywords: Bemotrizinol, Nanoemulsion, In vitro SPF, Ex-vivo deposition, Tape stripping technique, Photostability

(C) 2019 The Authors. Published by Innovare Academic Sciences Pvt Ltd. This is an open access article under the CC BY license (http://creativecommons.org/licenses/by/4.0/) DOI: http://dx.doi.org/10.22159/ijpps.2019v11i7.32652

\section{INTRODUCTION}

Many of our leisure activities take place outdoors, yet sun exposure has a negative impact on our skin, the effect of sunlight accumulate over a lifetime increasing the risk of developing permanent damage and consequently leads to skin cancer [1]. Sunscreen products are cosmetic products formulated to protect the skin from the damaged effects of solar radiations such as skin aging and skin cancer [2, 3] Sunscreens contain molecules or molecular complexes that can absorb, reflect, or scatter UV photons, in order to prevent, ameliorate, or even repair solar-induced skin damage, they can be classified according to their mode of action into: systemic and topical sunscreens. The latter have two types; inorganic or physical agents which show UV reflection, scattering and in some cases absorption e. g., titanium dioxide and zinc oxide [4] while organic or chemical agents which absorb high energy UV rays can be classified according to their UV spectrum into UVA absorbers, UVB absorbers and UVA+UVB absorbers (broad spectrum) e. g., bemotrizinol and bisoctrizole. Ideal sunscreen should be able to provide high protection effect to skin from harmful effect of UV rays and should be formulated in a cosmetically acceptable form [5].

Bemotrizinol is named as tinosorb $\mathrm{S}$, anisotriazine or Bisethylhexyloxyphenol methoxyphenyl triazene "BEMT" [6] and is considered as a hydrophobic sunscreening agent with high molecular weight $(>500 \mathrm{~g} / \mathrm{mol})$, broad spectrum sunscreen filter (280-380 nm) that does not penetrate the skin, because of its relatively large size mwt $627.8 \mathrm{~g} / \mathrm{mol}$. BEMT has fewer side effects and it rarely causes allergic contact dermatitis, systemic absorption, or endocrine-like effects and decreased skin permeability [7].

Nanoemulsion is thermodynamically stable transparent or translucent system of two immiscible liquids stabilized by an interfacial film of surfactant and co-surfactant with a droplet size less than $100 \mathrm{~nm}$ [8] and can be classified into three types: oil in water nanoemulsions (o/w) for lipophilic drug encapsulation, water in oil nanoemulsions (w/o) for hydrophilic drug encapsulation and bi-continuous nanoemulsions [9]. The advantages of loading bemotrizinol in $\mathrm{o} / \mathrm{w}$ nanoemulsion includes: a very small size of the nanoemulsion droplets which prevents any flocculation, creaming and sedimentation which might happens during storage; a good wettability, fluidity and spreadability; a transparent nature which gives a pleasant aesthetic character; the reduction in the trans-epidermal water loss due to its ability to strengthen the barrier function of the skin finally the high lipophilic nature of bemotrizinol makes it an excellent candidate to be loaded into nanoemulsion system.

The aim of this study was to improve the solubility of bemotrizinol in a nanoemulsion cosmetic gel vehicle in order to improve its sunscreen efficacy adopting a simple production technique, in addition to the production of non-greasy formulae with low skin permeation.

\section{MATERIALS AND METHODS}

\section{Materials}

Bemotrizinol (BEMT); cremophor EL (crem EL) and cremophor RH 40 (crem RH40) were kindly donated as a gift from (BASF, Germany); wheat germ, rose and argan oil (All of analytical grade and used without further purification); labrafac PG, capryol 90, lauroglycol 90 and labrasol were given as a gift from (Gattefossé, Saint Priest, France); isopropyl myristate (IPM), tween 80, 40 and 20 was purchased from (Sigma Aldrich, Germany); ethanol and methylene chloride (HPLC grade); potassium dihydrogen phosphate, disodium hydrogen phosphate, sodium chloride, magnesium sulphate and 
calcium chloride (El-Nasr pharmaceutical Chemical Company, Cairo, Egypt); cellulose membrane with mwt cut off 12000 Daltons (SigmaAldrich, USA); double distilled water; helioscreen plates (North Sutton, $\mathrm{NH}, \mathrm{USA}$ ); newborn rat skin (animal house of Cairo University, Egypt).

\section{Methods}

\section{Preformulation studies}

\section{Saturated solubility of bemotrizinol in different vehicles}

Bemotrizinol solubility was determined in various oils (IPM, labrafac PG, capryol 90, lauroglycol 90, wheat germ oil, rose oil and argan oil), various surfactants (tween 80,40 and 20) as well as various cosurfactants (crem EL and crem RH 40). An excess amount of BEMT was allowed to dissolve in $3 \mathrm{ml}$ vehicle in $10 \mathrm{ml}$ capacity stoppered vials separately. The mixtures were vortexed to facilitate solubilization using vortex mixer (stuart, UK). The mixture vials were kept at 37 ${ }^{\circ} \mathrm{C} \pm 1.0$ in an isothermal shaker for $72 \mathrm{~h}$ to reach equilibrium. The mixtures were removed from the shaker and centrifuged for $30 \mathrm{~min}$ at $2500 \mathrm{rpm}$ to remove undissolved BEMT. The supernatants were filtered through a $0.45 \mu \mathrm{m}$ millipore filter and the drug concentration in the filtrate was determined using a UV spectrophotometer (UV$1601 \mathrm{PC}$, Shimadzu, Kyoto, Japan) at $\lambda \max 340 \mathrm{~nm}$ after appropriate dilution with either methylene chloride in case of the oily phase or ethanol in case of surfactants and cosurfactants [10].

\section{Screening for S/CoS ratio using percentage of water incorporation method}

The ratios of surfactant to co-surfactants were chosen to be 1:0,1:1, 2:1, and 3:1. These mixtures $(\mathrm{S} / \mathrm{CoS})$ were mixed with the oil phase to give the weight ratios of 50:50, water was added drop by drop and stirred using a magnetic stirrer until a homogeneous dispersion or solution was obtained. After each addition, the system was examined for the appearance and flow properties. The end point of the titration was the point where the solution becomes cloudy or turbid. The quantity of the aqueous phase required to make the mixture turbid was noted. The percentages of the different incorporated phases were then calculated. The same procedure was followed for the other S/CoS ratios [11].

\section{Construction of nanoemulsion pseudo-ternary phase diagrams}

A combination of $\mathrm{S}$ and $\mathrm{CoS}$ were prepared in the form of mixture with fixed ratio $S / \operatorname{CoS}_{\text {mix }} 3: 1 \mathrm{w} / \mathrm{w}$ according to the result of $\%$ water incorporation method and it was used as one component of the pseudo-ternary phase diagram in addition to water and oil phases. Phase diagrams were constructed by mixing oil, $\mathrm{S} / \operatorname{CoS}_{\text {mix }}$ and water at certain weight ratios into glass vials, the mixtures in the vials were mixed using vortex (stuart, UK) for 2-3 min then incubated at room temperature for $72 \mathrm{~h}$ till equilibrium. After equilibrium, the mixtures were assessed visually as NEs [12]. All these points were marked in the phase diagrams and the area covered by these points was considered as the NE region of existence.

\section{Factorial experimental design of the study}

Mixed levels full factorial experimental design $\left(2^{4}\right)$ were carried out in order to investigate the influence of formulation variables on nanoemulsion properties using Design-Expert ${ }^{\circledR}$ software. The design was set at four independent variables: oil type, oil, $\mathrm{S} / \mathrm{CoS}_{\text {mix }}$ and drug concentration. The measured responses (dependent variables) were particle size and two hours in vitro release (Q2h).

\section{Preparation of bemotrizinol loaded nanoemulsion formulae}

In order to prepare the drug loaded NEs, the appropriate oil, $\mathrm{S} / \mathrm{CoS}_{\text {mix }}$ weight ratios were weighed in glass vials, then $1 \%$ or $2 \%$ $\mathrm{w} / \mathrm{w}$ of BEMT was accurately weighed and added to the mixture, vortexed and then water was added drop by drop at room temperature and vortexing was continued for $5 \mathrm{~min}$, the resultant NEs were stored for $72 \mathrm{~h}$ at room temperature till equilibrium reached before further investigations [13].

\section{Thermodynamic stability of bemotrizinol nanoemulsion formulae}

The susceptibility of $1 \%$ and $2 \% \mathrm{w} / \mathrm{w}$ BEMT loaded nanoemulsions for metastable formulation were evaluated through the thermodynamic stability tests. The formulae were firstly centrifuged at $3500 \mathrm{rpm}$ for $30 \mathrm{~min}$. The formulae that didn't show any phase separations were subjected to heating-cooling cycle test. Three complete cycles, each cycle consisting of $24 \mathrm{~h}$ at $5^{\circ} \mathrm{C}$ followed by $24 \mathrm{~h}$ at $45^{\circ} \mathrm{C}$. Formulae that passed these circumstances and showed good stability were subsequently subjected to three freeze-thaw cycles test, each cycle consisting of $24 \mathrm{~h}$ at $25^{\circ} \mathrm{C}$ followed by $24 \mathrm{~h}$ at $-5^{\circ} \mathrm{C}$ [12].

Pharmaceutical evaluation for the different prepared bemotrizinol nanoemulsion formulae

\section{Visual inspection}

The prepared formulae were examined for color, optical clarity, fluidity, homogeneity and phase separation (syneresis).

\section{Drug content determination}

The test was carried out by dissolving known quantity ( $0.1 \mathrm{~g}$ ) of prepared BEMT nanoemulsion in $100 \mathrm{ml}$ volumetric flask containing solvent (1\% tween 80 sweat simulated (SSW) solution [14] $(\mathrm{pH}=5.5)$. This solution was left for $15 \mathrm{~min}$ with continuous stirring using a magnetic stirrer to ensure the drug was completely dissolved. The absorbance of this solution was measured by using UV spectrophotometer (UV-1601 PC, Shimadzu, Kyoto, Japan) at the predetermined $\lambda_{\max }$ of BEMT and the percentage of drug content in each NE was calculated.

\section{pH measurement}

The $\mathrm{pH}$ of $10 \% \mathrm{w} / \mathrm{w}$ NE loaded drug in aqueous solution was measured by $\mathrm{pH}$ meter (HANNA instrument, USA). Solutions were prepared by dissolving $1 \mathrm{~g}$ of each NE formula in $9 \mathrm{~g}$ of double distilled water using magnetic stirrer. The $\mathrm{pH}$ meter was calibrated before each use with buffer solution of $\mathrm{pH} 4.0,7.0$ and 10 [10].

\section{Test of spreadability}

Spreadability plays an important role in the patient compliance and help in uniform application of NE formulae to the skin. It mainly depends on the viscosity of the NE formulae, an increase in viscosity leads to decrease in spreadability. A sample of $0.5 \mathrm{~g}$ of each formula was placed between two slides of glass plates and left for about 5 min where no more spreading was expected by the help of known weight $(1 \mathrm{~kg})$, after that the diameter of speadability of the $\mathrm{NE}$ was measured [15].

\section{Refractive index $(\eta)$}

Each NE system was measured by Abbe refractometer (WAG-25, Shanghahai, China) by placing one part of the formulation on the slide in triplicate at $25^{\circ} \mathrm{C}[16,17]$. The refractive index of a medium measures the extent of interaction between electromagnetic radiation and the medium through which it passes.

\section{Particle size and size distribution analysis}

The average droplet size and polydispersity index (PDI) of the prepared nanoemulsions were determined using Malvern Zetasizer (Malvern, UK). $0.1 \mathrm{~g}$ of each formula was dispersed into $10 \mathrm{ml}$ of distilled water with gentle stirring. An aliquot of diluted nanoemulsion was transferred into the cell sample holder for droplets size analysis [18].

\section{Determination of zeta potential}

Zeta potential was measured using Malvern Zetasizer (Malvern, UK). The diluted nanoemulsions with distilled water at ratio 1: 100 were exposed to an electric field (1v).

\section{Non-birefringence examination under cross-polarized microscope}

Bemotrizinol NE formulae were subjected to examination under cross-polarized microscope to test the absence of birefringence to exclude the presence of liquid crystalline systems [19]. A small quantity of the sample was placed on a clean glass slide and examined under cross-polarized microscope (Olympus, Japan).

\section{Rheological properties and viscosity measurements}

The viscosity of the prepared NE formulae was determined using (Brookfield DV III cone and plate Viscometer, ultra programmable 
rheometer, USA) using spindle 52 at $25^{\circ} \mathrm{C}$. A certain weight of each formula $0.5 \mathrm{~g}$ was placed in the plate of the viscometer. The rpm was increased gradually in a suitable range to give torque values between 10-100 units within $15 \mathrm{~s}$ between each successive speed.

\section{In vitro-release studies of bemotrizinol nanoemulsion formulae}

In vitro release study of BEMT nanoemulsion formulae and coarse BEMT powder as control [20] were performed using a USP dissolution apparatus II (Hanson research, USA) with slight modification in $300 \mathrm{ml}$ sweat simulated solution $(\mathrm{pH}=5.5)$ containing $1 \%$ tween 80 to maintain the sink condition [10] kept at $37 \pm 0.5{ }^{\circ} \mathrm{C}$. The stirring rate was $100 \mathrm{rpm}$. $2 \mathrm{mg} / \mathrm{cm}^{2}$ of each prepared NE formulae and coarse powder were placed in semipermeable membrane (cellulose nitrate pore size $0.45 \mu \mathrm{m}$ ) which is tight carefully from both sides and was hanged to the shaft of dissolution apparatus from both sides and dipped into the bottom of dissolution vessel. Every $60 \mathrm{~min}$ for $6 \mathrm{~h}, 5 \mathrm{ml}$ samples were withdrawn and replaced with equal volume of fresh medium to maintain a constant volume. Analysis of samples was done for their BEMT content using UV spectrophotometer (UV-1601 PC, Shimadzu, Kyoto, Japan) at the predetermined $\lambda_{\max }$, the mean percentage of BEMT released across the membrane was plotted as a function of time, all experiments were run in triplicates and the results were expressed as the mean average values $\pm S D$.

\section{Pharmaceutical evaluation of the selected BEMT nanoemulsion} formulae

Based on the highest in vitro release, the smallest particle size and the highest desirability values obtained from Design-Expert ${ }^{\circledR}$ software, the selected BEMT nanoemulsion formulae (F6 and F14) were subjected to further investigation.

\section{In vitro measurement of sun protection factor}

The measurement of SPF value for each formula were done and calculated using a Labsphere UV 1000S ultraviolet transmittance analyzer (Labsphere, Inc., North Sutton, NH USA) at wavelength range 290-400 $\mathrm{nm}$ [21] through a sample placed on a helioscreen plate with the selected formula. Weigh and apply $2 \mathrm{mg} / \mathrm{cm}^{2}$ of selected NE formulae over the helioscreen plates which possess topography parameters totally in compliance with ISO 24443 method-Annex D method and to the Colipa rev. 2011 method. The application of each formula was done by the aid of micropipette and rubs it with a single finger-cot-coated then put the plate aside to dry for at least $20 \mathrm{~min}$ before measurements begin. At least three samples were prepared for each formula.

\section{Water resistant test}

The water-resistance component of the SPF test consists of alternating fresh water-immersion and drying procedures. The test was done by the sunscreen application then, the helioscreen plates were immersed in a shaking water bath containing $5 \mathrm{l}$ fresh water used to prevent a high concentration of dispersed sunscreen and possible re-adsorption at $23-32{ }^{\circ} \mathrm{C}[22,23]$ beside moderate activity in water done by adjusting it at $30 \mathrm{rpm}$, each followed by a $20 \mathrm{~min}$ rest/air dry period until the total water exposure time was reached. The test areas were air dried without toweling at the end of the last water-immersion period. The SPF values were measured before and after each immersion cycle which presents the protection retained after each water immersion [24]. A product will be claimed to be 'water resistant if its \%WRR is $>50 \%$, and 'very water resistant' or 'waterproof' if its \%WRR is $>80 \%$ after two 20 min immersions in water.

\section{HPLC method for detection and quantification of bemotrizinol}

An isocratic reversed phase (RP) validated HPLC method [25] with slight modifications followed by validation for these modifications was used for detection and quantification of BEMT. The apparatus used for HPLC analysis was Agilant 1260 infinity LC system equipped with quaternary pump, an auto-sampler unit and UV detector. Nova-pak C18 $(150 \mathrm{~mm} \times 3.9 \mathrm{~mm})$ column packed with a 4 $\mu \mathrm{m}$-size adsorbent as stationary phase. The mobile phase was composed of $100 \%$ ethanol and the flow rate was set at $1.5 \mathrm{ml} / \mathrm{min}$.
The detector was set at $340 \mathrm{~nm}$ and the injection volume was $20 \mu \mathrm{l}$ at room temperature. The retention time of BEMT recorded under the previous conditions was about $2.5 \mathrm{~min}$. The stock solution of BEMT $0.1 \mathrm{mg} / \mathrm{ml}$ was prepared by dissolving $10 \mathrm{mg}$ of BEMT in 100 $\mathrm{ml}$ ethanol. Validation of assay procedures was done regarding linearity, precision, LLOD and accuracy.

\section{Ex-vivo permeation and deposition of bemotrizinol NE}

\section{Ex-vivo permeation test}

The full body skin of newborn rat (one week-hairless rat) was obtained from the animal house of Cairo University and the experimental protocol was ethically approved by the Animal Care Committee, Faculty of pharmacy, Helwan University No (3) for $27 / 11 / 2016$. The skin subcutaneous tissue was removed surgically if present and the dermis side was wiped with isopropyl alcohol to eradicate adhering fat. $2 \mathrm{mg} / \mathrm{cm}^{2}$ of the prepared NE formula was spread over the surface of the newborn rat skin which was held on a glass cylinder tube of $2.5 \mathrm{~cm}$ diameter and held together by waterproof plaster and equally spaced plastic clips. This assembly was placed at the bottom of the dissolution vessel containing $300 \mathrm{ml}$ of $1 \% \mathrm{w} / \mathrm{v}$ tween 80 in phosphate buffer saline (PBS) solution $(\mathrm{pH}$ $=7.4$ ) [26]. The permeation test was carried out for six hours at $37 \pm 1$ ${ }^{\circ} \mathrm{C}$. The stirring rate was adjusted at a speed of $100 \mathrm{rpm}$. Every 60 min an aliquot of $5 \mathrm{ml}$ of the medium was withdrawn and replaced with equal volume of fresh medium.

The concentration of BEMT was analysed by the developed and validated RP-HPLC method at $340 \mathrm{~nm}$ using (Agilent 1260 HPLC system, USA). The cumulative amounts of permeated BEMT through the skin per unit surface area were calculated and subsequently used for plotting the profiles of the drug permeation per unit time (t). The rate of drug permeation at steady state (drug flux) (Jss) was presented by the slope of the linear portion of the plotted profiles, the permeability coefficient $(\mathrm{Kp})$ was calculated by dividing the JSS with initial concentration of the drug $\left(\mathrm{C}_{0}\right) \mu \mathrm{g} / \mathrm{ml}$ [27].

\section{Ex-vivo deposition and accumulation test (Tape stripping technique)}

Our experimental study was extended for investigating the possibility of drug accumulation beneath the skin layers [28] and that deposit in the surface of stratum cornum. After $6 \mathrm{~h}$ the skin surface was washed five times with distilled water and was dried using filter paper in order to remove excess drug. By the aid of pieces of adhesive tape, the skin was tape stripping 20 times, tape strips and skin specimens were placed each in ethanol followed by sonication in order to extract the deposited drug [29], after $3 \mathrm{~d}$ the samples of strips and skin were filtered with filter memberane of pore size $0.45 \mu \mathrm{m}$ before injected into HPLC column using the developed and validated RP-HPLC method at $340 \mathrm{~nm}$ (Agilent 1260 HPLC system, USA) to analyse the amount of BEMT accumulated into SC, epidermis and dermis.

\section{Photostability and photostability kinetic studies}

Two criteria were considered to evaluate the photostability of sunscreens; the first one is based on the area under curve index by the aid of HPLC analysis and the second one is the residual percentage of two protection factors $[30,31]$ both give a similar ranking of the sunscreens photostability [6,32].

\section{Area under curve index}

Photodegradation of optimum formulae (F6 and F14) were evaluated using (Agilent 1260 HPLC system, USA) in the form of AUC. Each sample of $2 \mathrm{mg} / \mathrm{cm}^{2}$ was weighed and placed on a helioscreen plates $(4 \times 4 \mathrm{~cm})$. The sunscreen was distributed over the entire surface by slowly and deliberately rubbing the surface of the plate with a single finger-cot-coated. The samples were irradiated with UVA and UVB radiation of intensity 35.35 and $15.91 \mathrm{~W} / \mathrm{m}^{2}$ at different time intervals $20,40,60,80,100,120,140,160$ and 180 min while the control sample was kept in a dark place [33]. After exposure, each plate was transferred to a beaker and washed well with ethanol and subjected to sonication using (Sonicator, Crest ultrasonic Crop., New York, USA) to allow the complete extraction of 
the nanoemulsion from the surface of the plate, this was then transferred to a $100 \mathrm{ml}$ volumetric flask, diluted with ethanol to adjust volume [34]. The area under the curve of each sample was compared to AUC of both formulae before irritation (at zero time) [6, 33]. Each point was calculated using the following equation:

$$
\text { AUCIndex }=\frac{\text { AUC after }}{\text { AUC before }} \ldots . . . .(1)
$$

\section{Residual percentage of SPF}

SPF value after irradiation was compared to the in vitro SPF value before irradiation which was measured using Labsphere UV $1000 \mathrm{~S}$ ultraviolet transmittance analyzer (Labsphere, Inc., North Sutton, NH USA). The percentage variation of SPF [31] can be calculated as follow:

$$
\% \mathrm{SPF}=\frac{\text { In-vitro SPFafter }}{\text { In-vitro SPFbefore }} \times 100 \ldots . . .(2)
$$

Photostability kinetics was carried out by fitting the data of percentage SPF remaining after each time interval to different models namely; zero-order and first-order. The $t 90 \%$, and half-live $\left(\mathrm{t}{ }_{50 \%}\right)$ were donated as the time necessary to obtain a decrease of 10 and $50 \%$ of the initial SPF. The correlation coefficients $\left(\mathrm{r}^{2}\right)$ and photodegradation rate constant $(\mathrm{K})$ were also calculated. A time period of 120 min of irradiation was used to distinguish between good and poor photostability, because of the recommendation that a sunscreen is applied every $2 \mathrm{~h}$. This recommendation led us to classify the filters in two categories, those whose $t 90 \%$ is higher than $2 \mathrm{~h}$ (good photostability) and those whose $\mathrm{t} 90 \%$ is lower than $2 \mathrm{~h}$ (poor photostability) [33].

\section{RESULTS AND DISSCUSSION}

\section{Preformulation studies}

\section{Screening of bemotrizinol in different vehicle}

Based on the solubility study outcomes, labrafac PG and isopropyl myristate were used as oil, tween 80 and cremophor EL as surfactant and co-surfactant respectively for construction of the pseudoternary phase diagrams exhibiting the significantly highest BEMT solubility $(p>0.05)$ as shown in table 1 .

Table 1: Solubility of BEMT in different vehicles

\begin{tabular}{lll}
\hline Vehicle type & Vehicle & Solubility (mg/ml) \pm SD. \\
\hline \multirow{3}{*}{ Oil } & IPM & $129.47 \pm 0.46$ \\
& Labrafac PG & $86.75 \pm 2.86$ \\
& Capryol 90 & $57.77 \pm 0.22$ \\
& Wheat germ & $46.97 \pm 0.67$ \\
& Lauroglycol 90 & $36.39 \pm 1.20$ \\
& Rose oil & $34.67 \pm 0.09$ \\
Surfactant & Argan oil & $21.46 \pm 0.01$ \\
& Tween 80 & $42.89 \pm 0.02$ \\
Co-surfactant & Tween 20 & $19.67 \pm 0.88$ \\
& Tween 40 & $17.09 \pm 0.15$ \\
& Cremophore EL & $11.52 \pm 0.39$ \\
\hline
\end{tabular}

Data are presented as mean average value $\pm S D, n=3$.

Screening for $\mathrm{S} / \mathrm{CoS}$ ratio by using percentage of water incorporation method

The maximum water percentage incorporation (Wmax) of each ratio is presented in table 2 which showed that Wmax was remarkably high $(P<0.05)$ in ratio 3:1 in both oils. The Wmax of $S / C o S$ ratio was found in the following order 3:1>2:1 >1:1 >1:0. Increasing the $\mathrm{S} / \mathrm{CoS}$ ratio to $3: 1$ improved the micelle formation and increased the solubilizing capacity of the nanoemulsion, co-surfactant was inserted into the cavities between the surfactant molecules and consequently, increased the solubilizing capacity of the formed nanoemulsion to maximum value [35].

Table 2: Maximum water percentage incorporation of different $S / \operatorname{CoS}_{\text {mix }}$ ratios

\begin{tabular}{lll}
\hline S/CoS ${ }_{\text {mix }}$ ratios & Oil type & $\mathbf{W}_{\text {max }} \%(\mathbf{w} / \mathbf{w})$ \\
\hline Tween 80: Crem EL (1:0) & IPM & $18.44 \pm 0.065$ \\
& Labrafac PG & $10.12 \pm 0.075$ \\
Tween 80: Crem EL (1:1) & IPM & $21.80 \pm 0.055$ \\
& Labrafac PG & $13.38 \pm 0.29$ \\
Tween 80: Crem EL (2:1) & IPM & $23.07 \pm 0.11$ \\
& Labrafac PG & $15.62 \pm 0.14$ \\
Tween 80: Crem EL (3:1) & IPM & $26.97 \pm 0.2$ \\
& Labrafac PG & $16.34 \pm 0.17$ \\
\hline
\end{tabular}

Data are presented as mean average value $\pm S D, n=3$.

\section{Construction of nanoemulsion pseudo-ternary phase diagrams}

Six diagrams were developed for IPM and labrafac PG as oils, tween 80 as surfactant and crem EL as co-surfactant in fixed ratio (3:1) which exhibited the largest water incorporation percentage. Three diagrams were constructed for each oil; one diagram as blank base without drug, the other two diagrams one contains $1 \%$ BEMT and the second 2\% BEMT for each oil (fig. 1 and fig. 2).
These diagrams were drawn with the aid of Sigma plot software (version 13).

\section{Preparation of bemotrizinol loaded nanoemulsion formulae}

Eight formulae were prepared for each oil according to $2^{4}$ factorial design plan. The composition of different BEMT nanoemulsion formulae are given in table 3. 


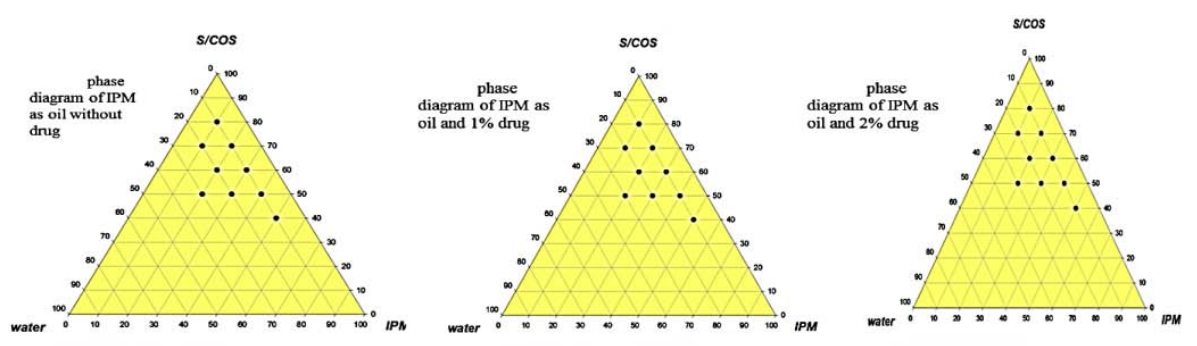

Fig. 1: Ternary phase diagram of IPM as oil

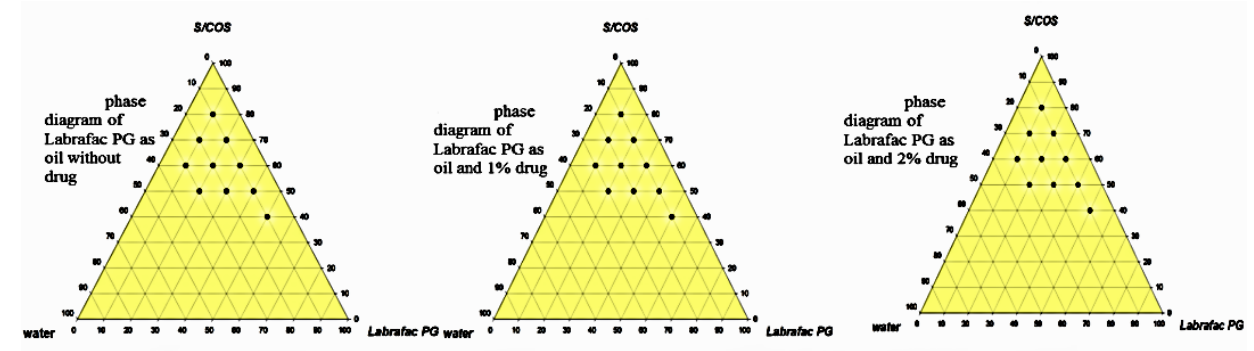

Fig. 2: Ternary phase diagram of Labrafac PG as oil

Table 3: Composition of the different prepared BEMT nanoemulsion formulae

\begin{tabular}{|c|c|c|c|c|c|c|c|c|}
\hline Composition (w/w \%) & F1 & F2 & F3 & F4 & F5 & F6 & F7 & F8 \\
\hline BEMT & $1 \%$ & $1 \%$ & $1 \%$ & $1 \%$ & $2 \%$ & $2 \%$ & $2 \%$ & $2 \%$ \\
\hline Labrafac PG & $20 \%$ & $20 \%$ & $35 \%$ & $35 \%$ & $20 \%$ & $20 \%$ & $35 \%$ & $35 \%$ \\
\hline $\mathrm{S} / \mathrm{CoS}_{\mathrm{mix}}$ & $50 \%$ & $60 \%$ & $50 \%$ & $60 \%$ & $50 \%$ & $60 \%$ & $50 \%$ & $60 \%$ \\
\hline Water & \multicolumn{8}{|c|}{ To $100 \mathrm{~g}$} \\
\hline Composition (w/w \%) & F9 & F10 & F11 & F12 & F13 & F14 & F15 & F16 \\
\hline BEMT & $1 \%$ & $1 \%$ & $1 \%$ & $1 \%$ & $2 \%$ & $2 \%$ & $2 \%$ & $2 \%$ \\
\hline IPM & $20 \%$ & $20 \%$ & $35 \%$ & $35 \%$ & $20 \%$ & $20 \%$ & $35 \%$ & $35 \%$ \\
\hline $\mathrm{S} / \mathrm{CoS}_{\operatorname{mix}}$ & $50 \%$ & $60 \%$ & $50 \%$ & $60 \%$ & $50 \%$ & $60 \%$ & $50 \%$ & $60 \%$ \\
\hline Water & \multicolumn{8}{|c|}{ To $100 \mathrm{~g}$} \\
\hline
\end{tabular}

Thermodynamic stability of bemotrizinol nanoemulsion formulae

None of the NE formulae showed phase separation or change in physical appearance such as turbidity or creaming. These results indicate that all NE formulae showed a good physical stability.

Pharmaceutical evaluation for the different prepared BEMT nanoemulsion formulae

\section{Visual inspection}

The prepared BEMT NEs showed yellowish clear homogeneous systems with gel consistency and no phase separation.

\section{Drug content determination}

The percentage drug content of NE formulae ranged from $98.85 \pm 0.32 \%$ to $107.4 \pm 0.2 \%$ as in table 4 . These results showed that the drug was uniformly distributed throughout the formulation and drug loss was low, these results indicated that the method of preparation was effective and of good capability to be loaded with BEMT up to $2 \% \mathrm{w} / \mathrm{w}$.

\section{pH measurement}

The $\mathrm{pH}$ of the prepared formulae was found to mimic the skin $\mathrm{pH}$ (5.5-7) [36] as shown in table 4.

\section{Test of spreadability}

The spreadability values of all formulae were found to be in the range of $4.9 \pm 0.08$ to $11.75 \pm 0.2 \mathrm{~cm}$ as shown in table 4 .

\section{Refractive index}

The mean values of the refractive index of the nanoemulsion formulae were found in the range of 1.3682 to 1.4604 which is a good indication that all prepared NEs formulae are transparent and optically isotopic [37] as shown in table 4 .

\section{Particle size and size distribution analysis}

The nanoemulsion average droplets were in size $<100 \mathrm{~nm}$ as shown in table 5 where the small size of the droplets might be attributed to the penetration of co-surfactant molecules into the surfactant film and its ability to reduce the surface viscosity, fluidity and droplet radius of curvature [38]. It seems that the mean particle size was decreased with less percentage of oil phase and high percentage of $\mathrm{S} / \mathrm{CoS}_{\text {mix }}$ [39]. Upon increasing the drug loading an increase in particle size was observed [40]. The polydispersity index (PDI) values were lower than 0.6 which indicate the uniformity of droplet size.

\section{Determination of zeta potential}

The zeta potential of all nanoemulsion formulae was found between$12.37 \pm 0.35 \mathrm{mV}$ to- $24.75 \pm 0.15 \mathrm{mV}$ as shown in table 5 . The zeta potential is a function of the surface charge which developed when any material is placed in a liquid. The negative charge indicates a deflocculated system in which repulsive forces exceeded the attractive forces, thereby keeping the particles dispersed [18].

\section{Non-birefringence examination under cross-polarized microscope}

Examination under cross polarized microscope showed that all prepared nanoemulsion formulae appeared dark, indicating isotropic properties of all formulae [41]. 
Table 4: Characterization of prepared nanoemulsion formulae

\begin{tabular}{|c|c|c|c|c|}
\hline Formulae code & Drug content (\%) & pH & Spreadability (cm) & Refractive index \\
\hline F1 & $101.73 \% \pm 0.15$ & $6.68 \pm 0.05$ & $5.70 \pm 0.08$ & $1.367 \pm 0.000$ \\
\hline $\mathrm{F} 2$ & $107.40 \% \pm 0.20$ & $6.82 \pm 0.01$ & $8.10 \pm 0.08$ & $1.447 \pm 0.000$ \\
\hline F3 & $104.75 \% \pm 0.05$ & $6.99 \pm 0.00$ & $7.60 \pm 0.08$ & $1.452 \pm 0.000$ \\
\hline F4 & $104.46 \% \pm 0.12$ & $6.26 \pm 0.00$ & $9.75 \pm 0.20$ & $1.452 \pm 0.001$ \\
\hline F5 & $104.67 \% \pm 0.64$ & $6.84 \pm 0.01$ & $5.90 \pm 0.08$ & $1.426 \pm 0.000$ \\
\hline F6 & $102.75 \% \pm 0.55$ & $6.27 \pm 0.01$ & $9.70 \pm 0.08$ & $1.447 \pm 0.000$ \\
\hline F7 & $106.36 \% \pm 0.33$ & $6.88 \pm 0.00$ & $11.75 \pm 0.20$ & $1.449 \pm 0.000$ \\
\hline F8 & $106.80 \% \pm 0.10$ & $6.33 \pm 0.00$ & $8.85 \pm 0.12$ & $1.460 \pm 0.000$ \\
\hline F9 & $100.27 \% \pm 0.18$ & $6.01 \pm 0.01$ & $7.20 \pm 0.04$ & $1.428 \pm 0.000$ \\
\hline F10 & $106.25 \% \pm 0.17$ & $6.95 \pm 0.04$ & $8.45 \pm 0.04$ & $1.444 \pm 0.000$ \\
\hline F11 & $105.88 \% \pm 0.41$ & $5.71 \pm 0.01$ & $8.95 \pm 0.04$ & $1.447 \pm 0.000$ \\
\hline F12 & $102.77 \% \pm 0.12$ & $7.00 \pm 0.00$ & $10.25 \pm 0.20$ & $1.455 \pm 0.000$ \\
\hline F13 & $102.61 \% \pm 0.33$ & $7.00 \pm 0.01$ & $4.90 \pm 0.08$ & $1.431 \pm 0.000$ \\
\hline F14 & $101.40 \% \pm 0.03$ & $6.36 \pm 0.01$ & $8.10 \pm 0.08$ & $1.448 \pm 0.000$ \\
\hline F15 & $106.30 \% \pm 0.29$ & $6.94 \pm 0.06$ & $8.40 \pm 0.08$ & $1.445 \pm 0.000$ \\
\hline F16 & $98.85 \% \pm 0.32$ & $6.81 \pm 0.00$ & $10.15 \pm 0.12$ & $1.460 \pm 0.000$ \\
\hline
\end{tabular}

Data are presented as mean average value $\pm S D, n=3$.

Table 5: Droplet size, PDI and zeta potential of prepared nanoemulsion formulae

\begin{tabular}{llll}
\hline Formula code & Droplet size (nm) & PDI & Zeta potential (mV) \\
\hline F1 & $27.23 \pm 0.60$ & $0.303 \pm 0.004$ & $-15.60 \pm 0.30$ \\
F2 & $18.83 \pm 0.30$ & $0.192 \pm 0.001$ & $-14.45 \pm 0.45$ \\
F3 & $44.24 \pm 1.64$ & $0.211 \pm 0.001$ & $-12.35 \pm 0.35$ \\
F4 & $31.41 \pm 0.83$ & $0.162 \pm 0.002$ & $-20.50 \pm 1.00$ \\
F5 & $57.40 \pm 0.77$ & $0.534 \pm 0.015$ & $-20.75 \pm 0.75$ \\
F6 & $41.00 \pm 10.00$ & $0.322 \pm 0.01$ & $-19.10 \pm 0.20$ \\
F7 & $166.30 \pm 1.60$ & $0.249 \pm 0.005$ & $-15.65 \pm 0.25$ \\
F8 & $62.90 \pm 0.73$ & $0.454 \pm 0.002$ & $-18.25 \pm 0.65$ \\
F9 & $49.28 \pm 0.81$ & $0.542 \pm 0.001$ & $-24.45 \pm 0.25$ \\
F10 & $24.16 \pm 0.99$ & $0.362 \pm 0.004$ & $-17.20 \pm 0.70$ \\
F11 & $47.00 \pm 1.00$ & $0.269 \pm 0.001$ & $-18.70 \pm 0.30$ \\
F12 & $35.11 \pm 1.31$ & $0.223 \pm 0.002$ & $-19.05 \pm 0.25$ \\
F13 & $54.50 \pm 1.50$ & $0.311 \pm 0.0035$ & $-24.75 \pm 0.15$ \\
F14 & $104.79 \pm 1.49$ & $0.495 \pm 0.018$ & $-20.60 \pm 0.50$ \\
F15 & $127.73 \pm 1.76$ & $0.284 \pm 0.005$ & $-17.55 \pm 0.25$ \\
F16 & $55.70 \pm 1.33$ & $0.381 \pm 0.004$ & $-20.15 \pm 0.15$ \\
\hline
\end{tabular}

Data are presented as mean average value $\pm S D, n=3$.

\section{Rheological properties and viscosity measurements}

BEMT NE formulae revealed non-newtonian, pseudo-plastic flow with thixotropy. The dermal dosage forms should exhibits comparatively high viscosity and plastic or thixotropic flow behavior to enable sticking onto the skin for a sufficient time [42].

\section{In vitro release studies of bemotrizinol nanoemulsion formulae}

As the manufacturers suggest that the consumers should apply sunscreen products every two hours to maintain a constant amount of UV-filter on the skin surface. The release of drug at $2 \mathrm{~h}$ was considered as a measure for analysis. The higher \% of drug released after $2 \mathrm{~h}$ was achieved by (F2, 6, 10 and 14) which might be attributed to their composition as they contain high concentration of $\mathrm{S} / \mathrm{CoS}_{\text {mix }}$ which allows the drug to be in a solubilized form and leads to small droplet size of their nanoemulsion making them excellent carrier for enhancing drug release. The lower \% of drug released after $2 \mathrm{~h}$ was exhibited in (F1, 5, 9 and 13) might be attributed to their high viscosity which delay or hinder the release of the drug from the formulation as shown in (fig. 3 and fig. 4).

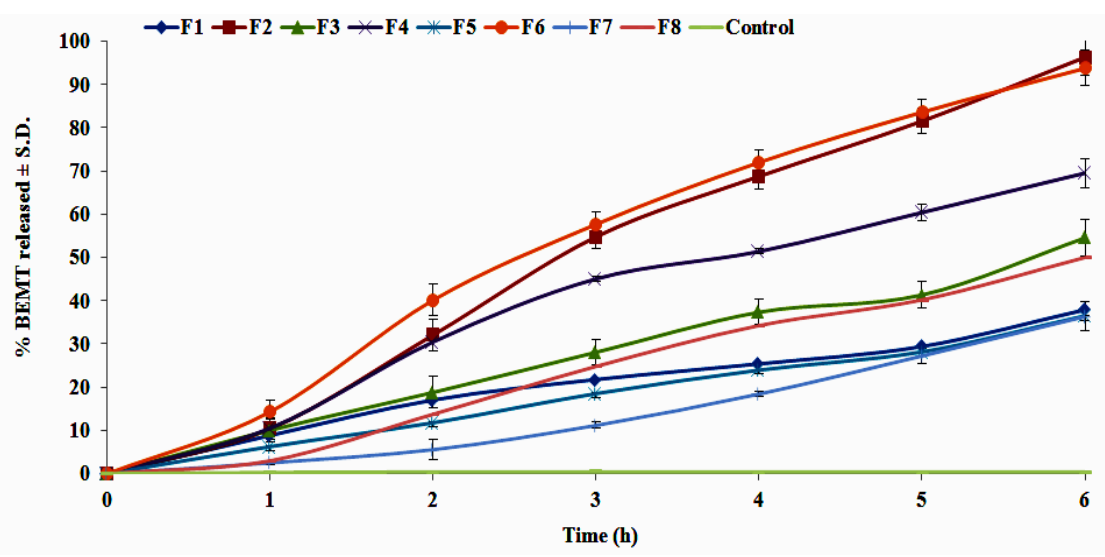

Fig. 3: Release pattern of BEMT from different prepared nanoemulsion formulae using Labrafac PG 


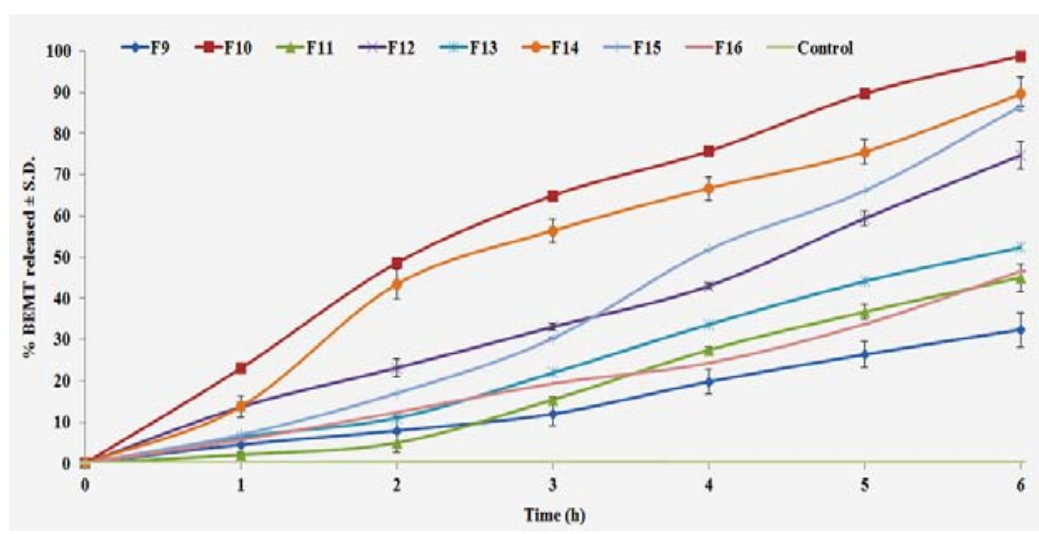

Fig. 4: Release pattern of BEMT from different prepared nanoemulsion formulae using IPM

\section{Statistical analysis of factorial design}

\section{Effect of formulation variables on particle size analysis}

The concentration of oil used has a statistically significant effect on particle size. Akshay R. Koli reported that increasing oil concentration increase particle size. This could be explained by the fact that the higher the oil phase concentration, the more viscous is the nanoemulsion since higher oil concentration increase the interfacial tension between the aqueous phase and oil phase. Increasing $\mathrm{S} / \mathrm{CoS}_{\text {mix }}$ concentration in nanoemulsion yields decreasing in droplet particle size due to increase the adsorption around the oil-water droplet interface and decreased interfacial tension in the system leading to form nanoemulsion with smaller droplets [8]. Increasing the drug concentration in nanoemulsion system leads to increasing the particle size as shown in (fig. 5) where the nanosize of $\mathrm{NE}$ droplets was affected with the incorporation of the drug. However, it remained within the NE range, the increase in droplet diameter is due to the embedding of the drug in the interfacial layer of surfactants, which might decrease the curvature of the micelle and thus change the size of the droplets [43]. The 3D surface plots of each couple of the three numeric factors are illustrated in (fig. 5).

\section{Effect of formulation variables on $2 \mathrm{~h}$ in vitro release}

Lower oil concentration resulted in higher \% BEMT released from nanoemulsion formulae. As oil concentration increased, bigger droplets were formed with larger mean particle size and hence a lower in the amount of drug released [44], this might be also attributed to the high affinity of drug to the vehicle [45]. The \% BEMT released from nanoemulsion formulae is higher in the presence of higher concentration of $\mathrm{S} / \mathrm{CoS}_{\text {mix }}$, this could be explained by the fact that small droplet size coupled with low interfacial tension [46]. Increasing the concentration of drug in drug loaded nanoemulsion leading to decrease in drug release across dialysis membrane, this is due to high concentration of drug leading to larger particle size so decrease in interfacial surface area for release of drug. The 3D surface plots of each couple of the three numeric factors are illustrated in (fig. 5).

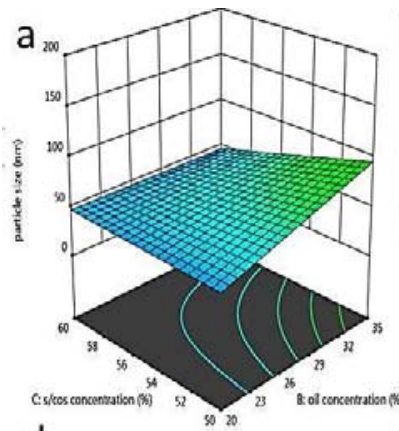

d
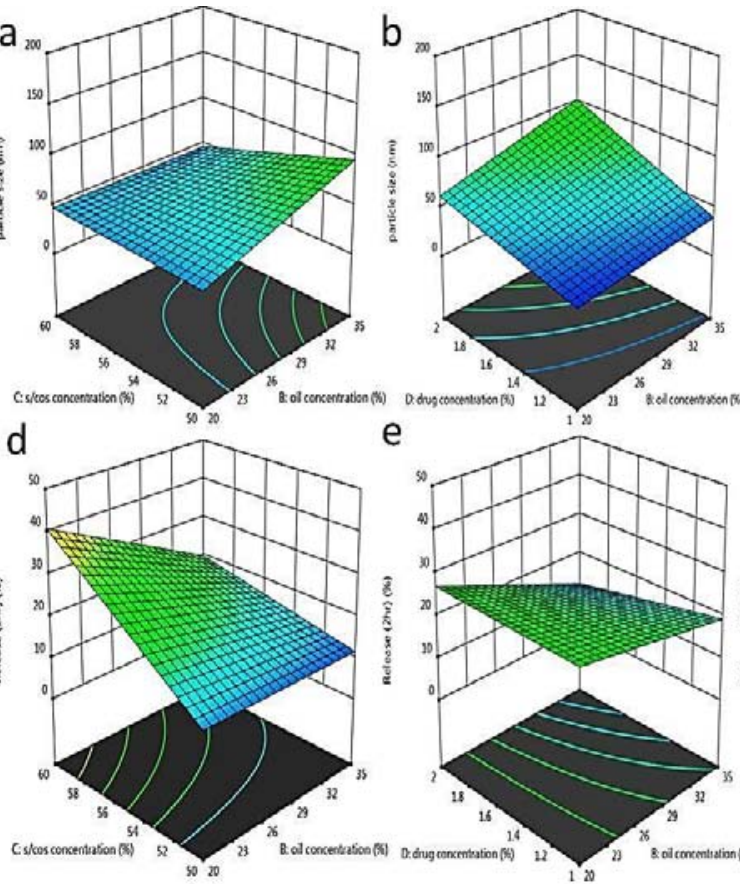

e

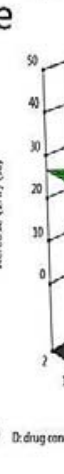

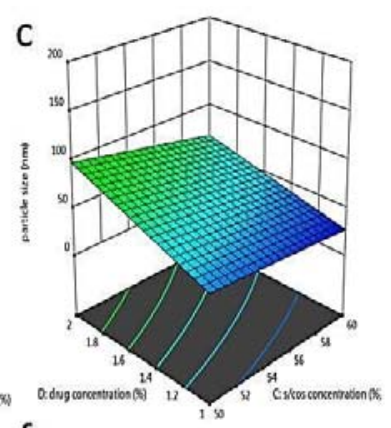

f

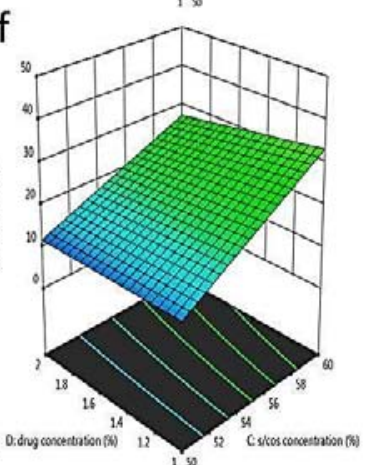

Fig. 5: Three dimensions surface plots for, a-The effect of oil concentration and $S$ /CoS concentration on particle size, $b$-The effect of oil concentration and drug concentration on particle size, $c$-The effect of $S / C o S$ concentration and drug concentration on particle size, $d$-The effect of oil concentration and S/CoS concentration on \% BEMT released after $2 \mathrm{~h}$, e-The effect of oil concentration and drug concentration on \% BEMT released after $2 \mathrm{~h}$, f-The effect of $\mathrm{S} / \mathrm{CoS}$ concentration and drug concentration on \% BEMT released after $2 \mathrm{~h}$ 
Evaluation tests of the selected BEMT nanoemulsion formulae

Based on the desirability value determined by Design-Expert ${ }^{\circledR}$ software, two nanoemulsion formulae were selected (F6 and F14 respectively) with desirability factor $(0.805,0.775)$ respectively.

\section{In vitro measurement of sun protection factor}

The formula containing IPM as oil (F14 SPF $=16.08 \pm 0.39$ ) showed noteworthy increase $\left(^{* * *} p<0.0001\right)$ than that contained labrafac PG (F6 $\mathrm{SPF}=10.30 \pm 0.12$ ). This might be due to higher solubility of the drug in the NE vehicle containing IPM rather than that containing labrafac PG.

\section{Water resistant test}

Results of the in vitro water resistant testing of the optimum BEMT NE formulae (F6 and F14) after 40 and 80 min water immersion were found to be the same and considered to be non-water resistant formulae as they were completely washed out from the helioscreen plates during the first $40 \mathrm{~min}$.

\section{Ex-vivo permeation and deposition of BEMT NE formulae (Tape} stripping technique)

The ex-vivo permeation parameters of F6 and F14 were illustrated in table 6. Upon comparing the permeation outcomes of the two selected NE formulae, a non-significant difference $(p>0.05)$ between them was found and they could considered as safe formulae since the maximum skin penetration in organic filters is up to $5 \%$ [47]. This low permeation result could be explained considering the high "substantivity" of bemotrizinol towards keratin, intended as the ability of a substance to be adsorbed or absorbed by keratin substrates of epidermis, thus resulting in a minimization of its percutaneous absorption [48], in addition to its high molecular weight [49] and high solubility in labrafac PG oil and IPM oil. Since the vehicle could increase the solubility of the drug, then it would be retained in it after application on the surface of skin, resulting in a reduced skin penetration [45]. After the end of permeation experiment, the efficacy of the nanoemulsion formulae to penetrate through the skin layers was determined $[29,49]$. The deposited and accumulated amount in newborn rat skin after exposing to BEMT nanoemulsion formulae were presented in table 7 . It was found that BEMT nanoemulsion formulae showed higher drug deposited in stratum cornum than that accumulated in epidermis and dermis. This high amount might be attributed to the components of both nanoemulsion formulae which could interact with the lipid bilayers of the stratum corneum, thus enhancing drug deposition [50].

Table 6: Permeation parameters of the selected BEMT nanoemulsion formulae after $6 \mathrm{~h}$

\begin{tabular}{lllllll}
\hline Formula & $\mathbf{r}^{2}$ & Flux $(\mathrm{Jss})\left(\boldsymbol{\mu g} / \mathbf{c m}^{\mathbf{2}} / \mathbf{h}\right)$ & $\mathbf{K p}(\mathbf{c m} / \mathbf{h})$ & $\mathbf{t}_{\mathbf{5 0} \%}(\mathbf{h})$ & Amount permeated $\left(\boldsymbol{\mu g} / \mathbf{c m}^{2}\right)$ \\
\hline F6 & 0.95 & 25.28 & 0.541 & 24.42 & 142.64 & Permeated $\%$ \\
F14 & 0.88 & 28.96 & 0.620 & 21.87 & 140.31 & 5.03 \\
\hline
\end{tabular}

Table 7: Ex-vivo deposition and accumulation data of selected BEMT nanoemulsion formulae

\begin{tabular}{lllc}
\hline $\begin{array}{l}\text { Formula } \\
\text { code }\end{array}$ & $\begin{array}{l}\text { Amount of BEMT deposited in } \\
\text { stratum cornum per unit area } \\
\left(\boldsymbol{\mu g} / \mathbf{c m}^{2}\right) \pm \mathbf{S D}\end{array}$ & $\begin{array}{l}\text { Amount of BEMT accumulated in } \\
\text { epidermis and dermis per unit area } \\
\left(\boldsymbol{\mu g} / \mathbf{c m}^{2}\right) \pm \text { SD }\end{array}$ & $\begin{array}{l}\text { \% deposited in } \\
\text { stratum cornum }\end{array}$ \\
\hline F6 & $681.902 \pm 26.54$ & $86.04 \pm 0.929$ & $\begin{array}{l}\text { \%accumulated in } \\
\text { epidermis and dermis }\end{array}$ \\
F14 & $643.034 \pm 43.39$ & $151.86 \pm 4.197$ & $23.91 \%$ \\
\hline
\end{tabular}

Data are presented as mean average value $\pm S D, n=3$.

\section{Photostability and photostability kinetic studies}

The results of area under curve index and residual percentage of SPFs gave a nearby finding of the sunscreens photostability [31, 33] after UV-light exposure at different time intervals as illustrated in table 8. Sunscreen was considered photostable when the percentage of SPF remained at least $80 \%$. A time period of $120 \mathrm{~min}$ of irradiation was used to distinguish between good and poor photostability $[31,34]$. From our findings the \% SPF remained after 120 min for $\mathrm{F} 6$ and $\mathrm{F} 14$ were $84.82 \%$ and $91.42 \%$ respectively, which means that the two selected nanoemulsion formulae possessed a good photostability.

Table 8: \% Recovery and \% SPF remained of selected BEMT nanoemulsion formulae at different time interval

\begin{tabular}{llll}
\hline Time (min) & \% Recovery of BEMT \pm SD & & \% SPF remained \pm SD \\
\cline { 2 - 4 } & F6 & F14 & F6 \\
\hline 0 & $100.00 \pm 0.20$ & $100.00 \pm 0.07$ & $100.00 \pm 0.45$ \\
20 & $96.89 \pm 0.20$ & $97.05 \pm 0.07$ & $95.97 \pm 0.77$ \\
40 & $93.83 \pm 0.27$ & $93.20 \pm 0.04$ & $91.50 \pm 0.84$ \\
60 & $91.53 \pm 0.46$ & $91.93 \pm 0.00$ & $89.50 \pm 1.11$ \\
80 & $87.35 \pm 0.02$ & $91.67 \pm 0.03$ & $85.34 \pm 0.38$ \\
100 & $85.23 \pm 0.16$ & 91.550 .026 & $85.32 \pm 0.28$ \\
120 & $85.06 \pm 0.13$ & $90.64 \pm 0.01$ & $84.82 \pm 0.62$ \\
140 & $83.88 \pm 0.12$ & $85.72 \pm 0.04$ & $82.06 \pm 0.28$ \\
160 & $82.19 \pm 0.06$ & $83.33 \pm 0.03$ & $80.05 \pm 0.12$ \\
180 & $80.53 \pm 0.12$ & $80.18 \pm 0.33$ & $77.67 \pm 0.17$ \\
\hline
\end{tabular}

Data are presented as mean average value $\pm S D, n=3$.

The gradual decrease in SPF during photodegradation of BEMT in the selected nanoemulsion formulae was expressed by its degradation rate constant $(\mathrm{K})$ which was calculated from the slope of the line of SPF versus time, $\mathrm{t} 90 \%, \mathrm{t} 50 \%$ and correlation coefficient $\left(\mathrm{r}^{2}\right)$ could be derived from kinetic analysis of the data as illustrated in table 9. According to the sunscreen stability testing, a product could be considered stable if it could preserve $80 \%$ of its effectiveness. In the event of exposure, it is recommended to the users to apply a sunscreen every two $h$. This recommendation led us to classify the filters in two categories, those whose $\mathrm{t} 90 \%$ is higher than two $\mathrm{h}$ (good photostability) and those whose $\mathrm{t}_{90 \%}$ is lower than two h (poor photostability) [51]. 
Table 9: Kinetic parameters outcomes of photostability of BEMT from F6 and F14 nanoemulsion formulae

\begin{tabular}{|c|c|c|c|c|c|c|c|c|c|}
\hline \multirow[t]{2}{*}{ Formula code } & \multicolumn{4}{|c|}{ Zero-order } & \multicolumn{4}{|c|}{ First-order } & \multirow[t]{2}{*}{ Order of degradation } \\
\hline & $\mathbf{R}^{\mathbf{2}}$ & $\mathbf{K}$ & $\mathbf{T}_{\mathbf{9 0} \%}$ & $\mathbf{T}_{\mathbf{5 0} \%}$ & $\mathbf{R}^{2}$ & $\mathbf{K}$ & $\mathbf{T}_{\mathbf{9 0} \%}$ & $\mathbf{T}_{\mathbf{5 0} \%}$ & \\
\hline F6 & 0.949 & 0.11 & 83.72 & 418.60 & 0.960 & 0.0006 & 81.75 & 537.80 & First-order \\
\hline F14 & 0.958 & 0.05 & 165.67 & 828.36 & 0.962 & 0.962 & 168.02 & 1105.41 & First-order \\
\hline
\end{tabular}

The $\mathrm{t} 90 \%$ was found to be equal to 81.75 and 168.02 min for F6 and F14 respectively which indicated that the optimum BEMT nanoemulsion formula was F14 where its $t_{90 \%}$ is higher than two $h$ when compared to F6. The high photostability of bemotrizinol was reckoned on its molecular structure that facilitates the dissipation of incident energy by intramolecular heat transfer and vibrational relaxation; therefore, no reactive intermediate species and no photolytic decomposition products were formed in the skin [52].

\section{CONCLUSION}

The developed bemotrizinol nanoemulsion showed good characterization in terms of rheological behavior making it suitable for skin application. The transparent non-gritty appearance of the prepared nanoemulsion confirmed the homogeneity dispersion of the drug in the system with improved filter efficacy by the aid of a single sunscreening agent, decreased permeation and enhanced photostability emphasize the potential use of nanoemulsions as cosmetic bases for lipophilic chemical sunscreening bemotrizinol.

\section{ACKNOWLEDGMENT}

Authors would like to acknowledge National Organization for Drug Control and Research for providing all facilities during project work.

\section{AUTHORS CONTRIBUTIONS}

Authors state that they didn't receive any payment in the preparation of this manuscript. Prof. Dr. Wedad Sakran and Dr. Vivian Elwazzan chose the topic and reviewed the scientific paper. Dr. Nermin Sheta and Dina Hashim implemented the idea in the lab and wrote the scientific paper.

\section{CONFLICT OF INTERESTS}

No conflict of interest was declared by the authors

\section{REFERENCES}

1. Haluza D, Simic S, Moshammer H. Sun exposure prevalence and associated skin health habits: results from the austrian population based uv skin risk survey. Int J Environ Res Public Health 2016;13:141.

2. Godwin D, Kim N, Felton L. Influence of transcutol CG on the skin accumulation and transdermal permeation of ultraviolet absorbers. Eur J Pharm Biopharm 2002;53:23-7.

3. Jimenez $M$, Pelletier J, Bobin M, Martini $M$. Influence of encapsulation on the in vitro percutaneous absorption of octyl methoxycinnamate. Int J Pharm 2004;272:45-55.

4. Sambandan D, Ratner D. Sunscreens: an overview and update. J Am Acad Dermatol 2011;64:748-58.

5. Latha M, Martis J, Shobha V, Sham R, Bangera S, Krishnankutty $\mathrm{B}$, et al. A review sunscreening agents. Clin Aesthet Dermatol J 2013;6:16-26.

6. Puglia C, Damiani E, Offerta A, Rizza L, Tirendi G, Tarico S, et al. Evaluation of nanostructured lipid carriers (NLC) and nanoemulsions as carriers for uv-filters: characterization, in vitro penetration and photostability studies. Eur J Pharm Sci 2014;51:211-7.

7. Shaath NA. SPF boosters and photostability of ultraviolet filters antioxidants and other cosmetics ingredients to improve the uv filters and boost the SPF. HAPPI; 2007. p. 77-83.

8. Zhang $\mathrm{Y}$, Shang $\mathrm{Z}$, Gao $\mathrm{C}, \mathrm{Du} \mathrm{M}, \mathrm{Xu} \mathrm{S}$, Song $\mathrm{H}$, et al. Nanoemulsion for solubilization, stabilization and in vitro release of pterostilbene for oral delivery. AAPS PharmSciTech 2014;15:1000-8.

9. Devarajan V, Ravichandran V. Nanoemulsions: as modified drug delivery tool. pharmacie globale. Int J Comprehensive Pharmacy 2011;4:1-6.
10. Badawi A, Abd El-Aziz N, Amin M, Sheta N. Topical benzophenone-3 microemulsion based gels: preparation, evaluation and determination of microbiological uv blocking activity. Int J Pharm Pharm Sci 2014;6:562-70.

11. Nornoo A, Osborne D, Chow D. Cremophore free intravenous microemulsions for paclitaxel. formulation, cytotoxicity and hemolysis. Int J Pharm 2008;349:108-16.

12. Ali H, Hussein A. Oral nanoemulasion of candesartan cilexetil: formulation, characterization and in vitro drug release studies. AAPS PharmSciTech 2017;3:1-16.

13. Yadav S, Poddar S. Formulation, in vitro and in vivo evaluation of nanoemulsion gel for transdermal drug delivery of nimodipine. Asian J Pharm Clin Res 2015;8:119-24.

14. Marques M, Loebenberg R, Almukainzi M. Simulated biological fluids with possible application in dissolution testing. Dissolution Technologies 2011;15-28. dx.doi.org/10.14227/ DT180311P15.

15. Kumar S, Aparna C, Raju B. Formulation and evaluation of losartan potassium nanoemulsion gel. Eur J Biomed Pharm Sci 2018;5:579-89.

16. Azeem A, Rizwan M, Ahmed F, Iqbal Z, Khar R, Aqil M, et al. Nanoemulsion components screening and selection: a technical note. AAPS PharmSciTech 2009;10:69-76.

17. Pathan I, Setty C. Stability evaluation of tamoxifen citrate nanoemulsion containing cremophor RH40 as surfactant. Acta Pharm Sci 2011;53:127-34.

18. Zhao Y, Wang C, Chow AH, Ren K, Gong T, Zhang Z, et al. Selfnanoemulsifying drug delivery system (SNEDDS) for oral delivery of zedoary essential oil: formulation and bioavailability studies. Int J Pharm 2010;383:170-7.

19. Sahoo S, Panyam J, Prabha S, Labhasetwar V. Residual polyvinyl alcohol associated with poly (D, L-lactide-coglycolide) nanoparticles affects their physical properties and cellular uptake. J Controlled Release 2002;82:105-14.

20. Alsofany J, Hamza M, Abdelbarry AA. Fabrication of nanosuspension directly loaded fast-dissolving films for enhanced oral bioavailability of olmesartan medoxomil: in vitro characterization and pharmacokinetic evaluation in healthy human volunteers. AAPS PharmSciTech 2018;19:2118-32.

21. Donglikar M, Deore S. A review article: sunscreens. Pharmacogn J 2016;8:171-9.

22. Pissavini M, Alard V, Heinrich U, Jenni K. In vitro assessment of water resistance of sun care products: a reproducible and optimized in vitro test method. Int J Cosmet Sci 2007; 29:451-60.

23. Stokes R, Diffey BL. In vitro assessment of sunscreen photostability: the effect of radiation source, sunscreen application thickness and substrate. Int J Cosmet Sci 1999;21:341-51.

24. Monteiro M, Ozzetti R, Vergnanini A, Gitirana L, Volpato N, Freitas $\mathrm{Z}$, et al. Evaluation of octyl p-methoxycinnamate included in liposomes and cyclodextrins in anti-solar preparations: preparations, characterizations and in vitro penetration studies. Int J Nanomed 2012;7:3045-58.

25. Stephane P. Stability of cosmetic formulations containing UV filters and preservatives, based on physical and chemical parameters. MOJ Toxicol 2015;1:12-21.

26. Ruela A, Perissinato A, Lino M, Mudrik P, Pereira G. Evaluation of skin absorption of drugs from topical and transdermal formulation. Braz J Pharmsci 2016;52:527-44.

27. Baboota S, Shakeel F, Ahuja A, Ali J, Shafig S. Design, development and evaluation of noval nanoemulsion formulations for transdermal potential of celecoxib. Acta Pharm 2007;57:315-32.

28. Junyaprasert V, Teeranachaideekul V, Souto EB, Boonne P, Muller RH. Q10 loaded NLC versus nanoemulsions: Stability, 
rheology and in vitro skin permeation. Int J Pharm 2009;377:207-14.

29. Bsieso E, Nasr M, Sammour O, Abd EL, Gawad N. Could nanovesicles containing a penetration enhancer clinically improve the therapeutic outcome in skin fungal diseases? Nanomed J 2015;10:2017-31.

30. Garoli D, Pelizzo M, Bernardini B, Nicolosi P. Sunscreen tests: correspondence between in vitro data and values reported by the manufacturers. J Dermatol Sci 2008;52:193-204

31. Hojerova J, Medovcikova A, Mikula M. Photoprotective efficacy and photostability of fifteen sunscreen products having the same label SPF subjected to natural sunlight. Int J Pharm 2011;408:27-38.

32. Kockler J, Oelgemöller M, Robertson S, Glass B. Photostability of sunscreens. J Photochem Photobiol C 2012;13:91-110.

33. Badawi A, Abd El-Aziz N, Amin M, Sheta N. Almond oil based oleogels as cosmetic bases for liposoluble sun-screening agent bemotrizinol: in vitro characterization and photostability. Inventi Rapid Cosmeceuticals 2015;4:175-85.

34. Gonzalez H, Tarras Wahlberg N, Stromdahl B, Juzeniene A, Moan J, Larko 0, et al. Photostability of commerical sunscreens upon sun exposure and irradiation by ultraviolrt lamps. BMC Dermatol 2007;7:1-9.

35. Chen H, Chang X, Weng T, Zhaox X, Gao Z, Yang Y, et al. A study of microemulsion systems for transdermal delivery of triptolide. J Controlled Release 2004;98:427-36.

36. Mahesh B, Vasanth K, Gowda D, Srivastava A, Raghundan H, Shinde $\mathrm{C}$, et al. Enhanced permeability of cyclosporine from a transdermally applied nanoemulgel. Der Pharm Sin 2015;6:69-79.

37. Archita P, Anita L. Self microemulsifying drug delivery system as a potential drug delivery system for protease inhibitors in the treatment of AIDS. Asian J Pharm Sci 2011;6:226-40.

38. Elmataeeshy M, Sokar M, Baheyeldin M, Shaker D. Enhanced transdermal permeability of terbinafine through novel nanoemulgel formulation, development, in vitro and in vivo characterization. Future J Pharm Sci 2018;4:18-28.

39. Moghimipour E, Salimi A, Karami M, Isazadeh S. Preparation and characterization of dexamethasone microemulsion based on pseudoternary phase diagram. Jundishapur G Nat Pharm Prod 2013;8:105-12.

40. Sakeena M, Muthanna F, Abdullah G, Kanakal M. Formulation and in vitro evaluation of ketoprofen in palm oil esters nanoemulsion for topical delivery. J Oleo Sci 2010;59:223-8.

41. Nemichand S, Laxman S. Solubility enhancement of nebivolol by microemulsion technique. J Young Pharm 2016;8:356-67.

42. Illing $\mathrm{A}$, Unruh $\mathrm{T}$. Investigation on the flow behavior of dispersion of solid triglyceride nanoparticles. Int J Pharm 2004;284:123-31.

43. Figueiredo K, Neves J, Silva J, Freitas R. Phenobarbital loaded microemulsion: development, kinetic release and quality control. Braz J Pharm Sci 2016;2:251-63.

44. Mortazavi S, Pishrochi S, Jafari AZ. Formulation and in vitro evaluation of tretinooin microemulsion as a potential carrier for dermal drug delivery. IJJPR 2013;12:599-609.

45. Montenegro L, Carbone C, Puglisi G. Vehicle effects on in vitro release and skin permeation of octyl-methoxycinnamate from microemulsions. Int J Pharm 2011;405:162-8.

46. Shalviri A, Sharma A, Patel D, Sayani A. Low surfactant microemulsions for enhanced topical delivery of poorly soluble drugs. J Pharm Pharm Sci 2011;14:315-24.

47. Ruszkiewicz J, Pinkas A, Ferrer B, Peres TV, Tsatsakis A, Aschner M. Neurotoxic effect of active ingredients in sunscreen products, a contemporary review. Toxicol Rep 2017;4:245-59.

48. Varvaresou A. Percutaneous absorption of organic sunscreens. J Cosmet Dermatol 2006;5:53-7.

49. Tampucci S, Burgalassi S, Chetoni P, Monti D. Cutaneous permeation and penetration of sunscreens: formulation strategies and in vitro methods. Cosmetics J 2018;5:1-17.

50. Khurana S, Jain NK, Bedi PM. Nanoemulsion based gel for transdermal delivery of meloxicam: physic-chemical, mechanistic investigation. Life Sci 2013;92:383-92.

51. Couteau C, Faure A, Fortin J, Paparis E, Coiffard LJ. Study of the photostability of 18 sunscreens in creams by measuring the SPF in vitro. J Pharm Biomed Anal 2007;44:270-3.

52. Kullavanijaya P, Lim H. Photoprotection. J Am Acad Dermatol 2005;52:937-58. 